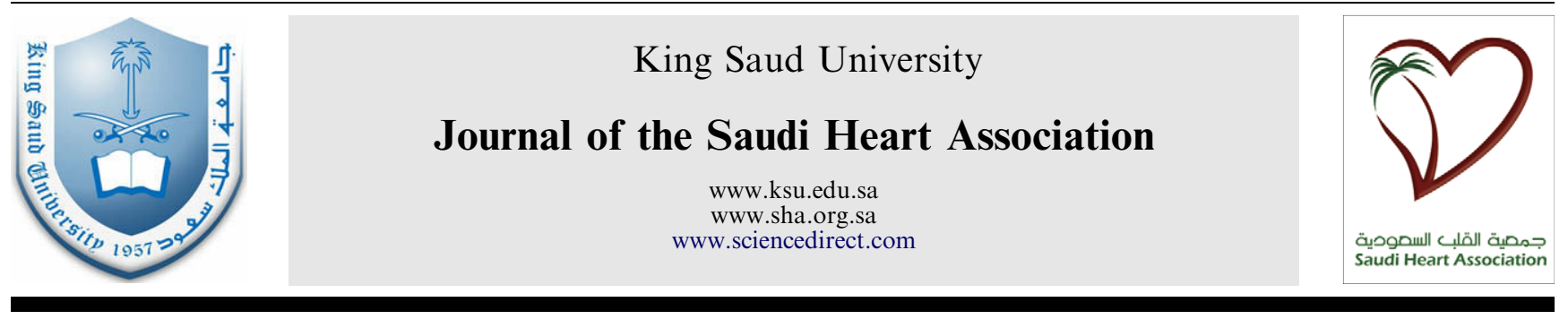

ORIGINAL ARTICLE

\title{
Hyperuricemia and its association with carotid intima-media thickness in hypertensive and non hypertensive patients
}

\author{
Abdelhakem Selem Elsayed ${ }^{\text {a,*, Mansour Mohamad Mostafa }}{ }^{\text {b }}$, \\ Alshazly Abdelkhalik ${ }^{\text {b }}$, Mohey Eldeen A. Eldeeb ${ }^{a}$, Mohammed Shafee Abdulgani ${ }^{\text {c }}$
}

${ }^{a}$ Department of Cardiology in Zagazig University, Egypt

${ }^{\mathrm{b}}$ Department of Cardiology in Al-Azhar University, Egypt

c Department of Cardiology, Aljedaani Hospital, Ibn Sina National School of Medicine, Jeddah, Saudi Arabia

Received 29 October 2009; accepted 30 December 2009

Available online 10 March 2010

\section{KEYWORDS}

Hyperuricemia;

Hypertension;

Left ventricular hypertrophy;

Caroid intima

mediathickness

\begin{abstract}
Carotid intima-media thickness (C-IMT) measured noninvasively by ultrasonography is widely used as a marker for increased risk of cardiovascular disease. Also hyperuricemia (HU) is a well recognized risk factor for cardiovascular diseases. The study was designed to assess the relation between hyperuricemia and carotid intima-media thickness C-IMT in patients with and without hypertension (HTN).

This study included 126 patients divided into four groups: (1) Group A, included 59 hypertensive patients with hyperuricemia. (2) Group B, included 29 hypertensive patients without hyperuricemia. (3) Group C, included 17 patients with hyperuricemia and normal blood pressure without history of hypertension. (4) Group D, included 21 control subjects.

We measured carotid intima-media thickness by B-mode ultrasound in the common carotid and internal carotid artery. Routine echocardiography and uric acid level was assessed for all patients.

We found that C-IMT was significantly higher in group A, B and C than group D; and it was significantly higher in group A than B. This means that C-IMT is significantly higher in all hypertensive groups than control group but it was significantly higher in hypertensive hyperuricemia (group A) than those hypertensives without hyperuricemia. We also observed a higher C-IMT in hyperuricemic non hypertensive patients than control group this means that hyperuricemia per se could be a risk factor for atherosclerosis.
\end{abstract}

\footnotetext{
* Corresponding author.

E-mail address: drhakeem66@yahoo.com (A.S. Elsayed).
}

1016-7315 @ 2010 King Saud University. All rights reserved. Peerreview under responsibility of King Saud University. doi:10.1016/j.jsha.2010.03.004

\begin{tabular}{ll}
\hline \\
ELSEVIER
\end{tabular}


Uric acid levels among the whole number of patients included in the study and among the groups with hyperuricemia (group A and group C) were positively correlated with the intimal-media thickness (IMT) while there were no correlations in the other two groups without hyperuricemia.

We found that left ventricular hypertrophy (LVH) was significantly higher in hypertensive patients (group A\&B) than normotensives (group C\&D) either with or without hyperuricemia and this was evident in the hypertensive hyperuricemic patients (group A); but unexpectedly we observed the presence of LVH in the hyperuricemic non hypertensive patients (group C) which was significantly higher than the control group (group D). This means that hyperuricemia is a risk factor for development of LVH hypertrophy independently of hypertension.

Therefore, higher serum uric acid levels are associated with increased C-IMT and left ventricular hypertrophy in hypertensive and even non hypertensive patients. So, early screening for hyperuricemia and lowering serum uric acid levels might be beneficial in slowing progression of atherogenesis.

(c) 2010 King Saud University. All rights reserved.

\section{Introduction}

It has been proven that the atherosclerotic changes in the carotid artery mirror general atherosclerosis (Baroncini et al., 2008). Carotid ntima-media thickness (C-IMT) is increasingly used in clinical trials as an important risk marker to investigate normal aging and preclinical atherosclerosis (Touboul et al., 2004). Several studies demonstrated that carotid IMT is significantly associated with risk for myocardial infarction, stroke, death from coronary artery disease, or a combination of these events (Stein et al., 2008; Culleton et al., 1999; Dawson et al., 2001; Ebrahim et al., 1999).

Hyperuricemia may be an independent risk factor associated with cardiovascular events (Alderman et al., 1999; Bickel et al., 2002; World Health Organization, 1999) and it is often considered as a part of metabolic syndrome or just a marker of other coronary risk factors such as hypertension, dyslipidemia, obesity or renal disease (Kanellis et al., 2003).

The possible contributory effect of hyperuricemia to carotid intima-media thickness (C-IMT) as marker of atherosclerosis in hypertensive as well as non hypertensive patients has not been clarified.

This study was designed to assess the relation between hyperuricemia and carotid intima-media thickness C-IMT in patients with and without hypertension (HTN).

\section{Patients and methods}

This study was carried out at Aljedani Hospital and Ibn Sina National Medical College in the Kingdome of Saudi Arabia from first of March 2009 to 31 August 2009. It included 126 patients divided into four groups: (1) Group A, included 59 hypertensive patients with hyperuricemia. (2) Group B, included 28 hypertensive patients without hyperuricemia. (3) Group C, included 17 non hypertensive patients with hyperuricemia. (4) Group D, included 21 control subjects non hypertensive with normal uric acid and they did not receive any medicine for hyperuricemia or hypertension in their past history. From the patient groups we excluded patient with systolic blood pressure of $220 \mathrm{~mm} \mathrm{Hg}$ or higher, acute coronary syndrome, stroke, or presence of a major illness such as cancer, liver disease, renal insufficiency and insulin-treated diabetes.

For all patients written consent, thorough history taking and clinical examination were done. Carotid intima-media thickness was measured by B-mode ultrasound in the common carotid and internal carotid artery from the thickest area along the course using Prosound Alfa 7 Aloka ultrasound machine with broad band linear probe having a frequency of $1-$ $15 \mathrm{MGz}$. And if there is significant stenosis by colored Doppler the velocity was taken in the stenosed area.

Routine echocardiography was done for all patients including left ventricular wall septal and posterior wall thickness, ejection fraction and fractional shortening. Uric acid level was assessed for all patients.

All data were categorized numerically, means and standard deviation; Pearson correlation and chi-square test were analyzed by using Excel Microsoft Office 2003. Significance was considered when $p$ value $<0.05$.

\section{Results}

For the demographic and clinical data there were no significant differences between all groups regarding age, sex and heart rate (Table 1).

C-IMT was significantly higher in group A, B and C than group $\mathrm{D} \quad(1.0 \pm 0.4 \mathrm{~mm}, \quad 0.92 \pm 0.28, \quad 0.91 \pm 0.17$ and $0.70 \pm 0.15$, respectively); and it was significantly higher in group A than B. This means that C-IMT is significantly higher in all hypertensive groups than control group but within hypertensive groups it was significantly higher in those with hyperuricemia (group A) than those hypertensives without hyperuricemia (group B). Also among non hypertensive patients C-IMT was higher in hyperuricemic group C than control group D.

Uric acid levels among the whole number of patients included in the study were positively correlated with C-IMT. Also uric acid levels in the groups with hyperuricemia (group A and group C) were positively correlated with C-IMT while there were no correlations in the other two groups without hyperuricemia (B and D) (see Figs. 1-5).

Left ventricular hypertrophy was significantly higher in hypertensive groups $(A \& B)$ than non hypertensive groups (C\&D), also It was significantly higher in group A (hypertensive hyperuricemic) than group B (hypertensive non hyperuricemic). While among the non hypertensive groups (A\&B) LVH was higher significantly in the hyperuricemic patients (group C) than control group D without hyperuricemia: this observation means that hyperuricemia per se has a relation to LVH in non hypertensive patients. 


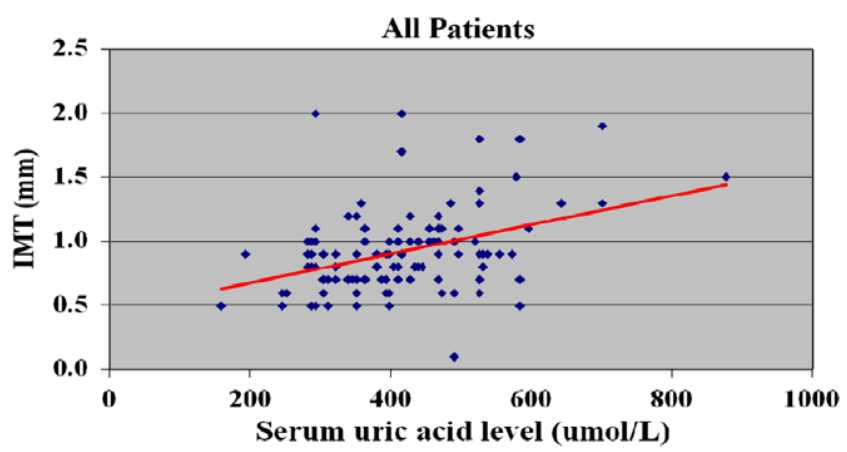

Figure 1 The correlation between serum uric acid level and CIMT in the whole number of patients.

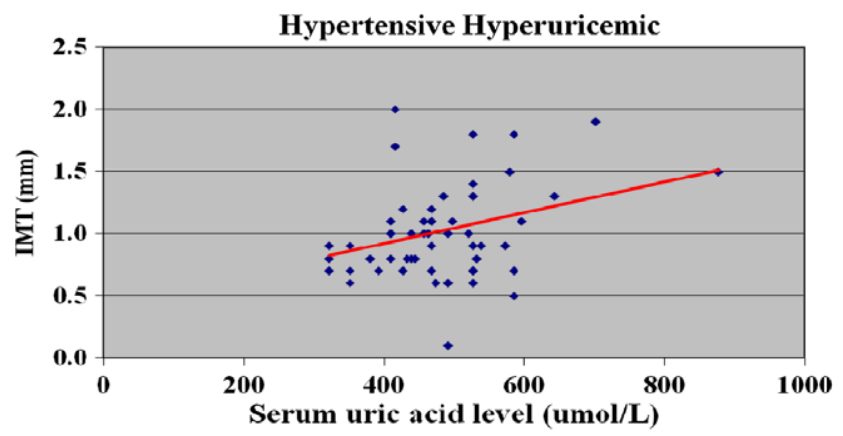

Figure 2 The correlation between serum uric acid level and CIMT in the hypertensive hyperuricemic group of patients (group A).

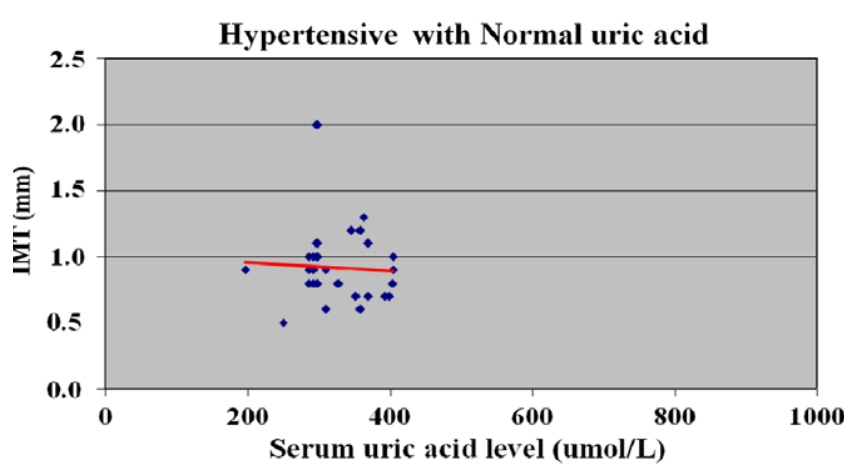

Figure 3 The correlation between serum uric acid level and CIMT in the hypertensive patients with normal uric acid (group B).

\section{Discussion}

The association between hyperuricemia and hypertension and the pathogenesis of atherosclerosis is still unclear. The present study was made in order to observe if hyperuricemia has a possible role in developing atherosclerosis in hypertensive and non hypertensive subjects.

In our study the control subjects (group D) who have the mean age of $40.5 \pm 9.0$; C-IMT was $0.70 \pm 15$ and this was similar to the normal value found in other study by Lim et al. (2008) who found that the normal value of the upper limits of C-IMT at the common carotid artery for the age 35-39, $40-49,50-59$, and 60 years or older were $0.60,0.64,0.71$, and $0.81 \mathrm{~mm}$, respectively, whereas for that at its bifurcation were $0.83,0.77,0.85$, and $1.05 \mathrm{~mm}$, respectively.

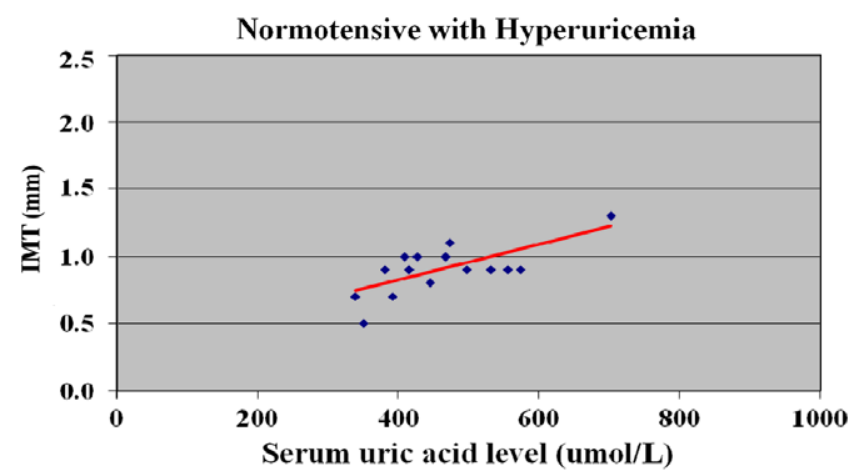

Figure 4 The correlation between serum uric acid level and CIMT in the normotensive hyperuricemic group of patients (group C).

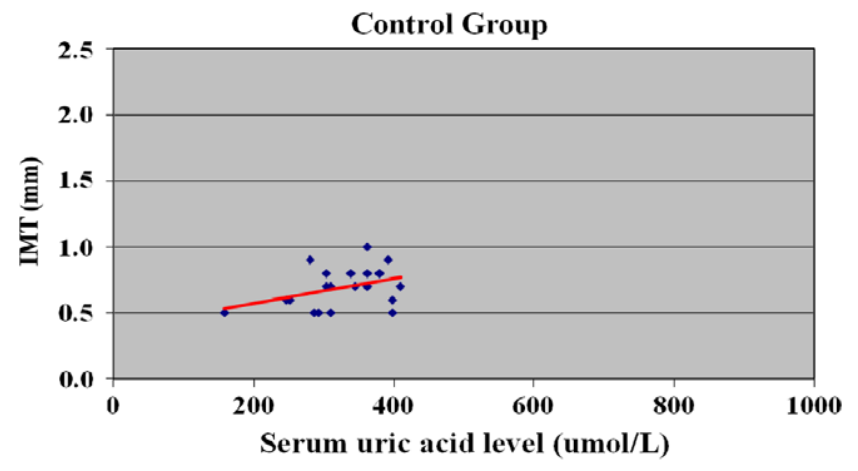

Figure 5 The correlation between serum uric acid level and CIMT in the control subjects (group D).

We observed that C-IMT is higher in patients with hypertension, with or without hyperuricemia, compared with the control group, while it was higher in hypertensive hyperuricemic than hypertensive non hyperuricemic patients. We also observed a higher C-IMT in hyperuricemic non hypertensive patients (group C) than control group (group D). This means that hyperuricemia per se could be a risk factor for atherosclerosis in both hypertensive as well as non hypertensive patients.

In consistent with our results Tavil et al. (2008) found that C-IMT was significantly higher in the hypertensive patients with or without hyperuricemia compared to the control cases $(0.70 \pm 0.14,0.83 \pm 0.16$ versus $0.57 \pm 0.16 \mathrm{~mm}$, respectively, $p<0.001$ ).

We noticed that there were significant correlations between C-IMT and serum uric acid levels among the whole number of subjects included in the study and this correlation was more evident in the hypertensive hyperuricemic (group A) and also in hyperuricemic normotensive (group C).

This result was similar in a study done by Savoiu et al. (2008) in 2008, who studied the effect of hyperuricemia among hypertensive patients but there study did not include the effect of hyperuricemia in the normotensive subjects.

We observed also that left ventricular hypertrophy $(\mathrm{LVH})$ was significantly higher in hypertensive patients (group A\&B) than normotensives (group C\&D) either with or without hyperuricemia and this was evident in the hypertensive hyperuricemic patients (group A); but unexpectedly we observed the presence of $\mathrm{LVH}$ in the hyperuricemic non hypertensive pa- 
Table 1 Clinical characteristics and biochemical parameters of the study subjects.

\begin{tabular}{lllll}
\hline & $\begin{array}{l}\text { Group A with HTN } \\
\text { and HU (no 59 pts) }\end{array}$ & $\begin{array}{l}\text { Group B with HTN } \\
\text { without HU (no 29 pts) }\end{array}$ & $\begin{array}{l}\text { Group C normotensives } \\
\text { with HU (no 17 pts) }\end{array}$ & $\begin{array}{l}\text { Group D control } \\
\text { group (no 21 pts) }\end{array}$ \\
\hline Age (years) & $51.98 \pm 8.89$ & $53.06 \pm 9.06$ & $64.7 \pm 11$ & $40.52 \pm 9.03$ \\
Male (\%) & 76.3 & 85.7 & 75 & 76.1 \\
Diabetes mellitus (\%) & 45.7 & 58.6 & 11.7 & 0 \\
Left ventricular hypertrophy (\%) & 57.6 & 51.7 & 41.1 & 0 \\
HDL-cholesterol (mg/dl) & $43.5 \pm 9.5$ & $42.0 \pm 9.4$ & $42 \pm 7.6$ & $48.9 \pm 7.1$ \\
LDL-cholesterol (mg/d) & $143.7 \pm 36.1$ & $132.6 \pm 47.8$ & $134.6 \pm 28.1$ & $104.7 \pm 20.1$ \\
Triglycerides (mg/dl) & $189.6 \pm 88.8$ & $179.5 \pm 54.9$ & $214.3 \pm 100.1$ & $142.9 \pm 49.8$ \\
Systolic blood pressure (mm Hg) & $161.0 \pm 17.1$ & $160.5 \pm 18.3$ & $125.6 \pm 11.8$ & $126.4 \pm 9.9$ \\
Diastolic blood $(\mathrm{mm} \mathrm{Hg})$ & $95.7 \pm 8.6$ & $93.3 \pm 9.09$ & $80.9 \pm 5.7$ & $79.04 \pm 6.8$ \\
Uric acid $(\mu \mathrm{mol} / \mathrm{l})$ & $478.7 \pm 94.5$ & $326.7 \pm 50.8$ & $461.4 \pm 91.1$ & $327.3 \pm 62.6$ \\
Carotid IMT $(\mathrm{mm})$ & $1.0 \pm 0.40$ & $0.92 \pm 0.28$ & $0.91 \pm 0.17$ & $0.70 \pm 0.15$ \\
\hline
\end{tabular}

tients (group C) which was significantly higher than the control group (group D). This means that hyperuricemia is a risk factor for development of LVH hypertrophy independently of hypertension.

However, many studies found the relation between LVH and high uric acid in hypertensive patients but scars reports studied this relation in those without hypertension.

In consistent with our study Mitsuhashi et al. (2009) found that hyperuricemia positively and independently associated left ventricular hypertrophy in Japanese non hypertensive men. In there study they as assessed LVH depending on electrocardiographic (ECG) criteria while in our study we assessed LVH by echocardiographic measurements of left ventricular posterior wall.

Kurata et al. (2005) reported that serum uric acid levels correlated positively with left ventricular mass and indexed left ventricular mass in male hypertensive patients but not in female hypertensive patients. Iwashima et al. (2006) also demonstrated an independent association between uric acid and left ventricular mass index and they suggested that the combination of hyperuricemia and LVH is an independent and powerful predictor of cardiovascular disease.

Many studies explained the effect of uric acids on LVH and endothelial dysfunction, it is reported to inhibit the generation of nitric oxide, thus inducing endothelial dysfunction and smooth muscle cell proliferation by activating inflammatory mediators such as tumor necrosis factor-alpha and mitogenactivated protein kinases (Iwashima et al., 2006; Khosla et al., 2005). UA is also capable of activating the rennin-angiotensin system, which is thought to mediate the development of LVH through many mechanisms (Agabiti-Rosei et al., 2006; Nakahara et al., 2007).

\section{Conclusion}

Carotid intimal media thickness C-IMT is increased in patients with hypertension as well as in patients with hyperuricemia without hypertension compared to the control group but it was more evident in hypertensive hyperuricemic patients.

Left ventricular hypertrophy is significantly higher in hyperuricemic patients than those without hyperuricemia especially in hypertensives as well as non hypertensives.

These results suggest that higher serum uric acid levels are associated with atherogenesis and left ventricular hypertrophy. Therefore, early screening for hyperuricemia and lowering ser- um uric acid levels might be beneficial in slowing progression of atherosclerosis in hypertensive and non hypertensive patients.

\section{References}

Agabiti-Rosei, E., Muiesan, M.L., Salvetti, M., 2006. Evaluation of subclinical target organ damage for risk assessment and treatment in the hypertensive patients: left ventricular hypertrophy. J. Am. Soc. Nephrol. 17, S104-S108.

Alderman, M.H., Cohen, H., Kivlighn, S., Madhavan, S., 1999. Serum uric acid and cardiovascular events in successfully treated hypertensive patients. Hypertension 34, 144-150

Baroncini, V.L.A., De Oliveira, A., Vidal, E.A., França, G.J., Sérgio Dalla, P., Stahlke, B., Alessi, A., Précoma, D.B., 2008. Appropriateness of carotid plaque and intima-media thickness assessment in routine clinical practice. Cardiovasc. Ultrasound 6 (52), 1186.

Bickel, C., Rupprecht, H.J., Blankenberg, S., Rippin, G., Hafner, G., Daunhauer, A., Hofmann, K.P., Meyer, J., 2002. Serum uric acid as an independent predictor of mortality in patients with angiographically proven coronary artery disease. Am. J. Cardiol. 89, 1217.

Culleton, B.F., Larson, M.G., Kannel, W.B., Levy, D., 1999. Serum uric acid and risk for cardiovascular disease and death: the Framingham heart study. Ann. Int. Med. 131, 7-13.

Dawson, J.D., Riley, W.A., Lauer, R.M., 2001. Carotid intimal-medial thickness is related to cardiovascular risk factors measured from childhood through middle age. The muscatine study. Circulation 104, 2815-2819.

Ebrahim, S., Papacosta, O., Whincup, P., et al., 1999. Carotid plaque, intima media thickness, cardiovascular risk factors, and prevalent cardiovascular disease in men and women: the British regional heart study. Stroke 30, 841-850.

Iwashima, Y., Horio, T., Kamide, K., Rakugi, H., Ogihara, T., Kawano, Y., 2006. Uric acid, left ventricular mass index, and risk of cardiovascular disease in essential hypertension. Hypertension $47,195-202$

Kanellis, J., Watanabe, S., Li, J.H., et al., 2003. Uric acid stimulates monocyte chemoattractant protein-1 production in vascular smooth muscle cells via mitogen-activated protein kinase and cyclooxygenase-2. Hypertension 41, 1287-1293.

Khosla, U.M., Zharikov, S., Finch, J.L., Nakagawa, T., Roncal, C., $\mathrm{Mu}, \mathrm{W}$., et al., 2005. Hyperuricemia induces endothelial dysfunction. Kidney Int. 67, 1739-1742.

Kurata, A., Shigematsu, Y., Higaki, J., 2005. Sex-related differences in relations of uric acid to left ventricular hypertrophy and remodeling in Japanese hypertensive patients. Hypertens. Res. 28, 133139. 
Lim, T.K., Lim, E., Dwivedi, G., Kooner, J., Senior, R., 2008. Normal value of carotid intima-media thickness - a surrogate marker of atherosclerosis: quantitative assessment by B-mode carotid ultrasound. J. Am. Soc. Echocardiogr. 21 (2), 112-116.

Mitsuhashi, H., Yatsuya, H., Matsushita, K., Zhang, H., Otsuka, R., Muramatsu, T., Takefuji, S., Hotta, Y., Kondo, T., Murohara, T., Toyoshima, H., Tamakoshi, K., 2009. Uric acid and left ventricular hypertrophy in Japanese men. Circ. J. 73, 667-672.

Nakahara, T., Takata, Y., Hirayama, Y., Asano, K., Adachi, H., Shiokawa, G., et al., 2007. Left ventricular hypertrophy and geometry in untreated essential hypertension is associated with blood levels of aldosterone and procollagen type III aminoterminal peptide. Circ. J. 71, 716-721.

Savoiu, G., Serban, C., Novanu, L., Mladinescu, F., Gaita, D., Duicu, O.M., Tudor, A., Muntean, D., Mihalas, G., 2008. The role of hyperuricemia in endothelial dysfunction induced by hypertension. Romanian J. Biophys. 18 (4), 329-336.
Stein, J.H., Korcarz, C.E., Hurst, R.T., Lonn, E., Kendall, C.B., Mohler, E.R., et al., 2008. ASE consensus statement. Use of carotid ultrasound to identify subclinical vascular disease and evaluate cardiovascular disease risk: a consensus statement from the American society of echocardiography. Carotid intima mediathickness task force endorsed by the society of vascular medicine. J. Am. Soc. Echocardiogr. 21, 93-111.

Tavil, Y., Kaya, M.G., Oktar, S.O., Sen, N., Okyay, K., Yazici, H.U., Cengel, A., 2008. Uric acid level and its association with carotid intima-media thickness in patients with hypertension. Atherosclerosis 197 (1), 159-163.

Touboul, P.J., Hennerici, M.G., Meairs, S., Adams, H., Amarenco, P., Desvarieux, M., et al., 2004. Mannheim intima-media thickness consensus. Cerebrovasc. Dis. 18, 346-349.

World Health Organization, 1999. International society of hypertension guidelines for the management of hypertension. J. Hypertens. 17, 151-183. 